\title{
Functional Dichotomy within the Vomeronasal System: Distinct Zones of Neuronal Activity in the Accessory Olfactory Bulb Correlate with Sex-Specific Behaviors
}

\author{
Arvind Kumar, Carol A. Dudley, and Robert L. Moss ${ }^{\dagger}$ \\ University of Texas Southwestern Medical Center at Dallas, Department of Physiology, Dallas, Texas 75235-9040
}

\begin{abstract}
Chemosensory neurons in the vomeronasal organ (VNO) detect pheromones that elicit social and reproductive behaviors in most terrestrial vertebrates. Vomeronasal receptor neurons are chemoarchitecturally divided into two populations based on their position in the VNO, the type of G-protein subunit expressed, the family of putative pheromone receptor expressed, and termination site of their axons in the accessory olfactory bulb (AOB). To investigate the functional implications of these two segregated VNO-AOB pathways, we stimulated mice with pheromonal cues associated with different behavioral contexts and examined cellular activation patterns in the AOB. Exposure of ICR male mice to BALB/c males resulted in aggressive behavior, accompanied by a VNO-dependent increase in c-fos immunoreactivity in a cluster of cells located almost exclusively in the caudal AOB in both strains. This caudal cluster of activated cells did not appear to require the overt display of ag-
\end{abstract}

gressive behavior because it was present in both the dominant and submissive males and could be evoked when the stimulus animal was anesthetized. In contrast, exposure of an ICR male to an ICR female in diestrus resulted in activation of cells located predominantly in the rostral AOB. Our findings indicate that male-to-male interactions involving interstrain recognition activate a separate population of vomeronasal receptor neurons than chemosensory cues detected in a sexual context. The results suggest that the dichotomy in the peripheral vomeronasal system serves to separate pheromones based on the behaviors they drive. As such, the results provide a bioassay for identifying pheromone molecules.

Key words: vomeronasal organ; accessory olfactory bulb; pheromones; sex-specific behaviors; c-fos immunoreactivity; mice
Pheromone signals are important for a wide variety of sociosexual functions, including puberty onset, estrous cycling, aggression, copulatory behavior, and recognition of reproductive state, gender, and strain (Wysocki, 1979; Yamazaki et al., 1981; MüllerSchwarze, 1983; Halpern, 1987; Vandenbergh, 1994; Døving and Trotier, 1998). A number of chemosensory systems could, in theory, mediate pheromonal influences on sociosexual functions, but in rodents, the olfactory and vomeronasal systems seem to be of primary importance. In the context of reproductive biology, the vomeronasal or accessory olfactory system plays a crucial role because lesions of the vomeronasal organ (VNO) or the accessory olfactory bulb (AOB) reduce the ability of the animal to respond to chemosensory cues both behaviorally (Wysocki, 1979) and in terms of reproductive endocrinology (Keverne, 1983).

The VNO contains two subdivisions of vomeronasal receptor neurons (VRNs) that are distinguished from one another by the family of putative pheromone receptor expressed (Herrada and Dulac, 1997; Matsunami and Buck, 1997; Ryba and Tirindelli, 1997), G-protein subunit content (Dulac and Axel, 1995; Halpern et al., 1995; Berghard and Buck, 1996), and termination site of their axons in the AOB (Jia and Halpern, 1996). These two populations of VNO neurons are anatomically segregated within the VNO into apical and basal zones. The apically located VRNs

\footnotetext{
Received May 26, 1999; revised July 29, 1999; accepted Aug. 12, 1999.

This research was supported by National Institutes of Health Grant MH41784. ${ }^{\dagger}$ Published posthumously.

Correspondence should be addressed to Carol A. Dudley, University of Texas Southwestern Medical Center at Dallas, Department of Physiology, 5323 Harry Hines Boulevard, Dallas, TX 75235-9040.

Copyright (C) 1999 Society for Neuroscience $\quad 0270-6474 / 99 / 190001-\bullet \$ 05.00 / 0$
}

coexpress $\mathrm{G}_{\alpha \mathrm{i}}$ and putative pheromone receptor V1R and project to several glomeruli in the rostral part of the AOB (Belluscio et al., 1999; Rodriguez et al., 1999), whereas the basally located VRNs express $\mathrm{G}_{\alpha \mathrm{o}}$, putative pheromone receptor $\mathrm{V} 2 \mathrm{R}$, and project to the caudal part of the AOB (Fig. 1a).

Although the projection patterns and signal transduction components of VRNs have been studied in some detail, the physiological significance of the dichotomy in the cytoarchitecture of the vomeronasal system is unknown. The two types of VRNs may detect different classes of ligands and/or may direct different aspects of pheromone-mediated physiology. To gain insight into the functional implications of these two segregated VNO-AOB pathways, we stimulated male mice with chemosensory cues associated with different behavioral contexts and examined cellular activation patterns within the AOB. In the context of reproductive behavior, exposure of male mice to female mice or female urine has been reported to result in a VNO-dependent increase

This article is published in The Journal of Neuroscience, Rapid Communications Section, which publishes brief, peerreviewed papers online, not in print. Rapid Communications are posted online approximately one month earlier than they would appear if printed. They are listed in the Table of Contents of the next open issue of JNeurosci. Cite this article as: JNeurosci, 1999, 19:RC32 (1-6). The publication date is the date of posting online at www.jneurosci.org.

http://www.jneurosci.org/cgi/content/full/3549 
in luteinizing hormone and testosterone (Wysocki et al., 1983; Coquelin et al., 1984) and to promote copulatory behavior (Clancy et al., 1984). In the context of aggressive behavior, removal of the VNO has been shown to decrease the display of aggression in male and lactating female mice (Clancy et al., 1984; Bean and Wysocki, 1989), whereas exposure to intact male urine or to constituents of male urine increased aggression in males (Novotny et al., 1985). Using induction of the c-fos protein as an index of cellular activation, we determined the location of activated cells in the AOB of male mice after stimulation by male- or female-associated chemosensory cues. c-Fos induction has been well documented to correlate with neuronal activity in the accessory olfactory system (Dudley et al., 1992; Fernandez-Fewell and Meredith, 1994; Bressler and Baum, 1996).

\section{MATERIALS AND METHODS}

Exposure to chemosensory stimuli. Adult ICR male, ICR female, and $\mathrm{BALB} / \mathrm{c}$ male mice were purchased from Charles River Laboratories (Wilmington, MA) and group housed in separate cubicles with food and water available ad libitum. In five ICR males, the vomeronasal organ was surgically removed (VNX), and three ICR males underwent sham surgery as described previously (Dudley and Moss, 1999). For exposure to chemosensory stimuli, pairs of animals were permitted $30 \mathrm{~min}$ direct contact with or $2 \mathrm{hr}$ close proximity to each other. For contact exposure, male mice of the ICR strain were placed in a shoebox lined with clean bedding and covered with a filter top for 10-15 min before the stimulus animal was introduced. The stimulus animal was an ICR male from a different cage, a BALB/c male, or an ICR female in diestrus. The pair of mice was allowed $15 \mathrm{~min}$ contact before the stimulus animal was returned to the home cage. After $45 \mathrm{~min}$, the stimulus animal was reintroduced for 15 min of contact. During male-male interactions, an observer scored the number of aggressive attacks during the $30 \mathrm{~min}$ of contact. Precautions were taken to prevent serious injury to the submissive male. In some cases, the BALB/c male was placed in the shoe box first and allowed to adapt before the introduction of an ICR male. To prevent the display of aggressive behavior, in some cases, the stimulus male was anesthetized with a mixture of ketamine, xylazine, and acepromazine. Anesthetized males were kept on a heating pad in between exposure periods and were allowed to fully recover. During male-female interactions, notations were made of any effort by the male to copulate with the unreceptive female. For noncontact exposure, a piece of wire mesh grid was folded in half and force-fit into a shoebox. An ICR or BALB/c male was placed on one side of the mesh for 10-15 min before the introduction of the stimulus animal on the other side. The pair of mice remained separated in this manner for $2 \mathrm{hr}$.

c-FOS immunocytochemistry. Two hours after the beginning of exposure, mice were overdosed with Nembutal (Abbott Laboratories, North Chicago, IL) and perfused with cold, phosphate buffered $3 \%$ paraformaldehyde. Immunocytohemical processing for c-fos and the method of dividing the AOB into rostral and caudal halves were as described previously (Dudley and Moss, 1999). In the present study, we measured the area of the rostral and caudal portions of the mitral and granule layer in three to six sections from three representative animals using the NIH Image program. The mean $\pm \mathrm{SE}$ area for the various portions of the two layers was as follows: rostral mitral layer, $0.100 \pm 0.006 \mathrm{~mm}^{2}$; caudal mitral layer, $0.084 \pm 0.005 \mathrm{~mm}^{2}$; rostral granule layer, $0.105 \pm 0.006$ $\mathrm{mm}^{2}$; and caudal granule layer, $0.096 \pm 0.003 \mathrm{~mm}^{2}$. Although the rostral areas were slightly larger than the caudal areas, matched-pair $t$ tests revealed that the difference was not significant. As seen in the histologically stained section in Figure $1 b$, the overall density of the cell bodies appears to be fairly even throughout the rostrocaudal extent of both the mitral and granule layers, but the width of the granule layer gradually decreases toward the caudal end.

c-Fos-immunopositive cells, recognized by the presence of a dark brown reaction product in the nucleus, were counted without knowledge of treatment from six sections of the AOB for each animal. Separate cell counts of the mitral and granule layers were made. Very few periglomerular cells were c-fos-immunopositive, and they were not included in the analysis.

Data analysis. Treatment effects on the total number of c-fosimmunopositive cells in each layer were analyzed by a one-way ANOVA, followed by post hoc Newman-Keuls multiple comparisons. To compare


Figure 1. $a$, Organization of the pathways from the VNO to the AOB. The photomicrograph at the top is a coronal section of the VNO in which medial is to the right and dorsal is at the top. Open triangles represent apical receptor cells expressing putative pheromone receptor V1R and $\mathrm{G}_{\alpha \mathrm{ai}}$, and open hexagons represent basal receptor cells expressing putative pheromone receptor $\mathrm{V} 2 \mathrm{R}$ and $\mathrm{G}_{\alpha \mathrm{o}}$. $b v$, Blood vessel. Underneath the VNO is a photomicrograph of a parasagittal section of the AOB showing localization of immunocytochemically detected $G_{\alpha i}$ in the rostral half of the glomerular layer $(\mathrm{glm})$. The line indicates the division between the rostral and caudal halves (see Materials and Methods). mit, Mitral cell layer; lot, lateral olfactory tract; grn, granule cell layer. In this and all subsequent photomicrographs, rostral (Rost.) is to the right and caudal (Caud.) is to the left. Scale bar (in this and all subsequent photomicrographs), $100 \mu \mathrm{m}$. $b$, Sagittal section of the mouse AOB stained with cresyl violet. Filled arrowheads point to mitral cell layer (enclosed by brackets), and open arrows point to the granule cell layer (enclosed by braces). The two layers of cell bodies are separated by the lateral olfactory tract.

the distribution of activated cells after various treatments, a one-way ANOVA on the difference between the number of activated cells located in the rostral AOB and the number of activated cells in the caudal AOB was performed for each layer, followed by post hoc Newman-Keuls multiple comparisons or $t$ tests. Chosen sections were photographed with 


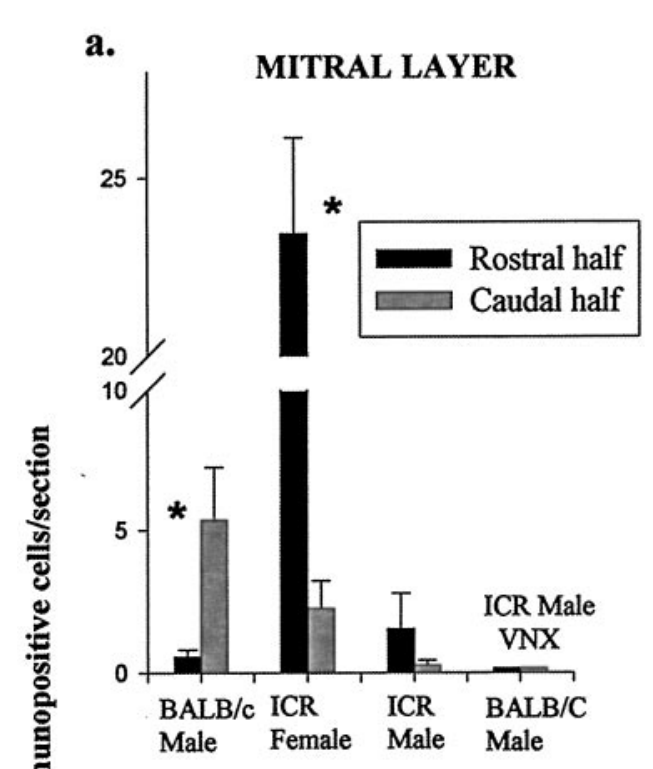

b.



c.

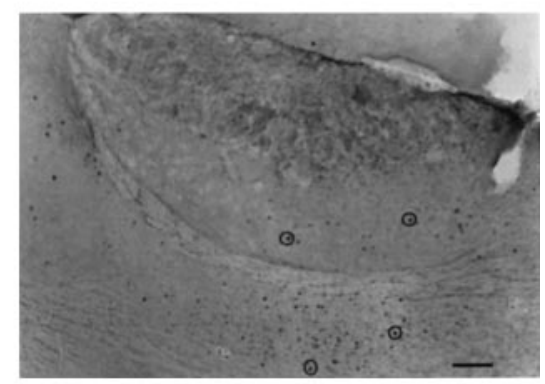

d.

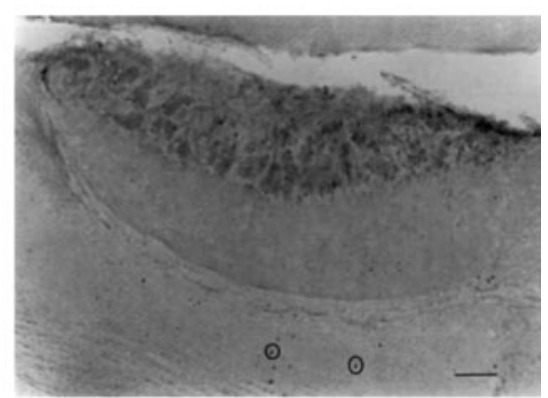

e.

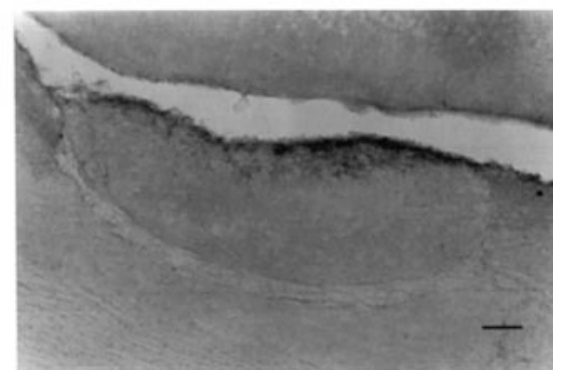

\section{Stimulus animal presented to ICR male}

Figure 2. Effect of exposure to various stimulus animals on the number of c-fosimmunoreactive cells and their distribution pattern in the mitral and granule cell layers of the ICR male mouse AOB. $a$, Histograms summarizing the total number and the rostrocaudal distribution of activated cells in the mitral (top) and granule (bottom) layers. Asterisks indicate a significant difference $(p<$ 0.05 ) between rostral and caudal activated cells within a given treatment group as revealed by matched-pair $t$ tests. $b-e$, Photomicrographs of representative parasagittal sections of the ICR male mouse AOB showing c-fos immunoreactivity after $30 \mathrm{~min}$ contact with a BALB/c male $(b)$, an ICR female in diestrus $(c)$, and an ICR male $(d)$. In $e$, the ICR male was VNX before exposure to a $\mathrm{BALB} / \mathrm{c}$ male. In $b-d$, the dark nuclear reaction product is circled for a few c-fos-positive cells. a Nikon (Tokyo, Japan) camera attached to a Leitz (Wetzlar, Germany) Ortholux II microscope. Prints were digitally scanned into PhotoDraw for labeling and sizing.

\section{RESULTS}

\section{Behavioral observations during contact exposure}

Direct exposure of intact ICR males to males of a different strain, i.e., BALB/c males, resulted in fighting. In every case, the ICR male rapidly displayed aggressive behavior, characterized by tail rattling and chasing, and attacking and biting the BALB/c male (mean $\pm \mathrm{SE}$ number of aggressive attacks, $55.2 \pm 8.5$ ), whereas the BALB/c male was submissive. Two of the ICR males that were VNX displayed aggressive behavior (mean, $53.5 \pm 23.5$ ), whereas the other three did not display aggressive behavior in the presence of a BALB/c male. ICR males paired with ICR males from a different cage sometimes exhibited aggressive behavior, but the intensity was much reduced compared with contact with a
BALB/c male $(n=5$; mean, $28.3 \pm 6.6)$. When paired with the ICR female in diestrus $(n=4)$, the ICR male vigorously sniffed the anogenital region of the female, usually attempting one or two mounts that were unsuccessful because the female was not receptive. The remainder of the exposure was characterized by intermittent sniffing and physical contact of a nonsexual nature.

\section{Patterns of cellular activation after contact exposure}

The histograms in Figure $2 a$ summarize the effect of exposure to the various stimulus animals on the total number of c-fosimmunoreactive cells and the rostrocaudal distribution of activated cells. There was a significant treatment effect on the total number of c-fos-immunopositive cells in both the mitral $(F=$ 30.26 ; $\mathrm{df}=2,13 ; p=0.00005)$ and granule $(F=14.97$; $\mathrm{df}=2,13$; $p=0.0009)$ layers. Post hoc comparisons indicated that contact with the ICR female in diestrus activated significantly more cells than contact with either the BALB/c male or the ICR male. 
a.

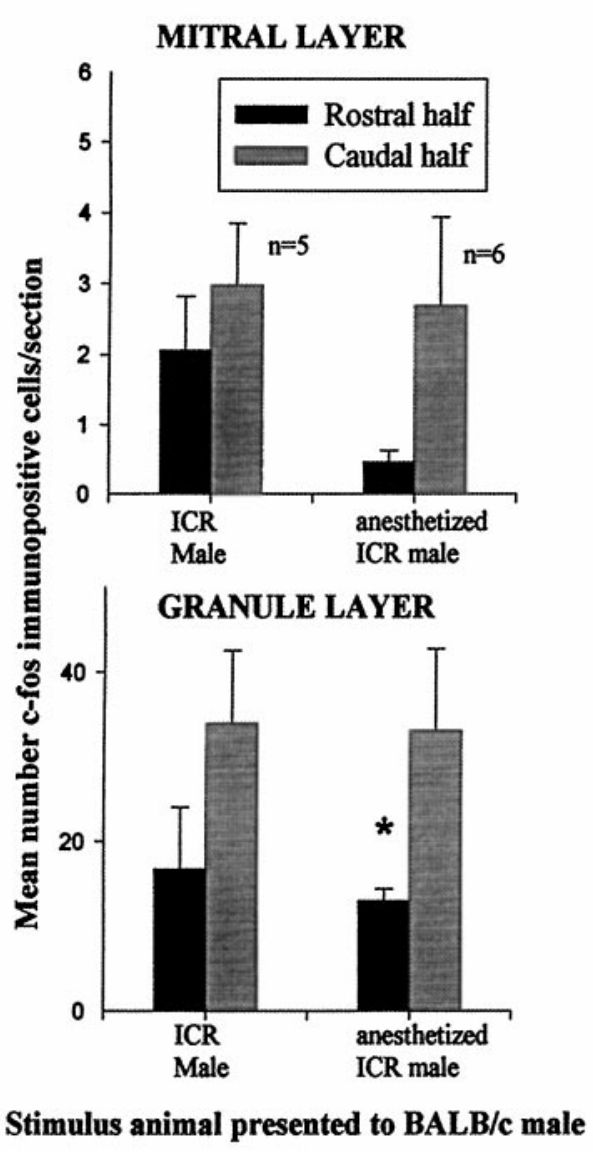

b.

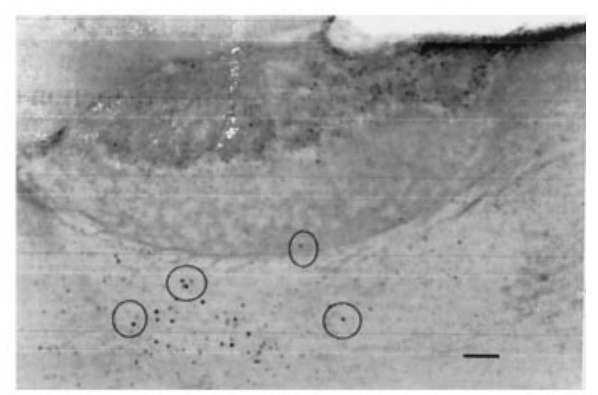

c.

d.
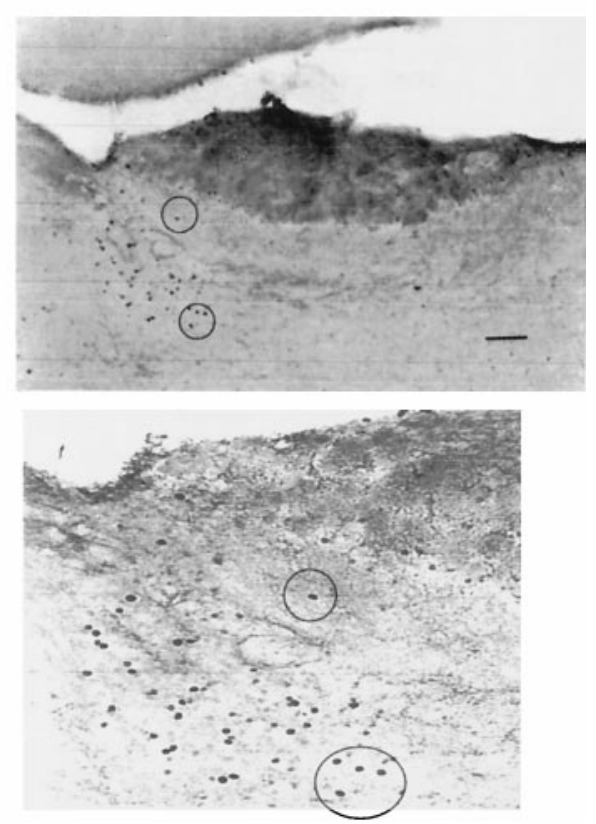

Removal of the VNO before exposure to a BALB/c male completely blocked c-fos induction in the AOB of ICR males. The c-fos response in sham-operated animals was similar to that of unoperated males (data not shown).

The rostrocaudal distribution of activated cells was significantly different for all three treatments (mitral layer, $F=55.38$; $\mathrm{df}=2$, $13 ; p=0.000004$; granule layer, $F=30.04$; $\mathrm{df}=2,13 ; p=$ $0.00005)$. Exposure to the ICR female produced cellular activation that was predominantly rostral, and the BALB/c male produced cellular activation that was predominantly caudal, whereas cellular activation in response to the ICR male was evenly distributed. The dramatic differences in the pattern of c-fos induction after exposure to the BALB/c male, the ICR female in diestrus, or the ICR male are illustrated in the photomicrographs in Figure $2 b-d$. Exposure to the BALB/c male produced cellular activation in a relatively small cluster of cells located in the caudal half of the AOB and sometimes was only observed at the extreme caudal end. In contrast, cellular activation evoked by the diestrus female was pronounced and heavily concentrated in the rostral AOB, gradually diminishing toward the caudal end. ICR maleto-ICR male contact resulted in a small number of activated cells that were fairly evenly distributed along the rostrocaudal axis. VNO removal resulted in degeneration of the glomerular layer of the AOB and the absence of c-fos-immunopositive cells (Fig. 2e).

Although the BALB/c males did not exhibit aggressive behavior, $\mathrm{c}$-fos induction in these males was still more prominent in the caudal AOB than in the rostral part (Fig. 3). Conversely, in pairings between ICR males when aggressive behavior was seen, no specific activation was observed in the caudal AOB. Also, no cellular activation was observed in the two VNX males exhibiting aggressive behavior. These findings suggest that the chemosensory cues activating the caudal AOB are related to strain recognition and not to the overt display of aggressive behavior. Compared with the ICR aggressor (Fig. 2), however, the caudal dominance in activated cells in the submissive BALB/c males was diminished, especially in the mitral layer. Thus, the overt display of aggressive behavior might be an important component in the activation of the caudal AOB. Therefore, we exposed BALB/c males directly to anesthetized ICR males for $30 \mathrm{~min}$. In this situation, the BALB/c males did not display aggressive behavior toward the anesthetized ICR males, yet c-fos activation was again confined to the caudal AOB (Fig. 3), suggesting that aggression is not required for this response.

\section{Pattern of activation after noncontact exposure}

We have reported previously a prominent, VNO-dependent c-fos activation in the rostral AOB of ICR male mice exposed to soiled bedding from cycling females (Dudley and Moss, 1999) and have more recently shown that urine alone, presented on filter paper, was also effective (Dudley et al., 1999). These findings indicate that c-fos activation in the rostral AOB is not dependent on physical contact with the female. However, the possibility remained that direct contact with the urine was required. To investigate the nature of the chemosensory cues responsible for 


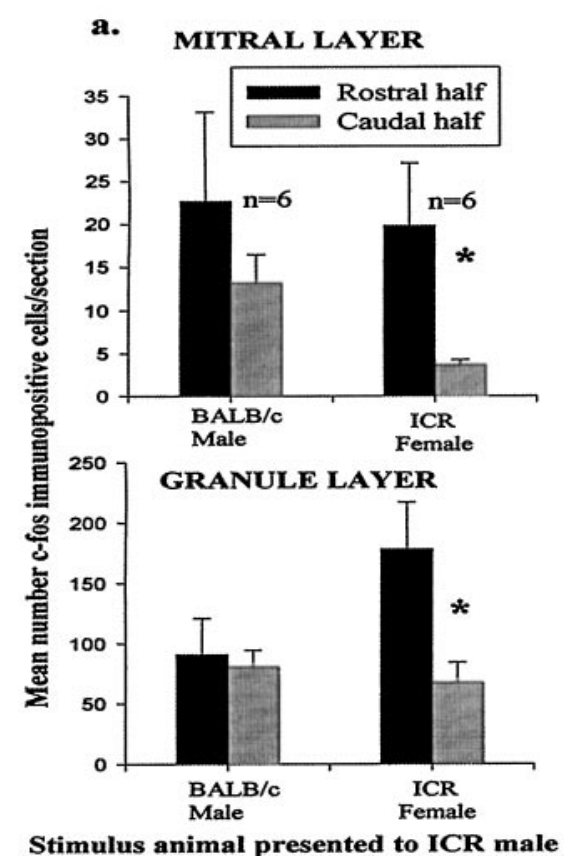

b.

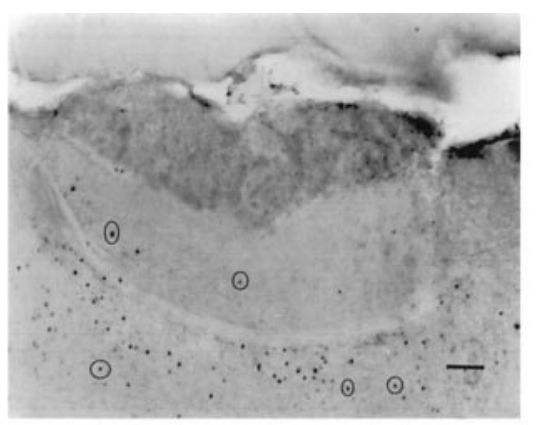

c.

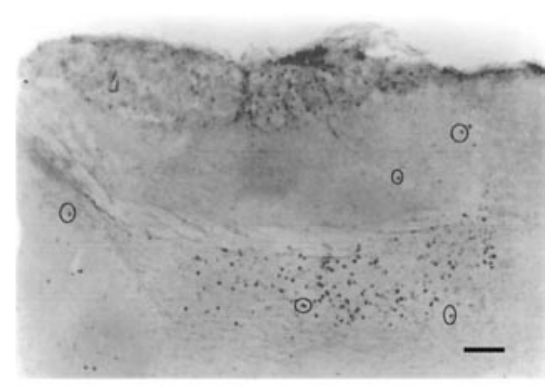

Figure 4. Cellular activation in the AOB of the ICR male mouse after $2 \mathrm{hr}$ barrier separation from the indicated stimulus animal. $a$, Histograms summarizing the total number and the distribution of c-fos-immunopositive cells in the mitral and granule layers. The predominantly caudal activation observed after contact exposure to the BALB/c male (Fig. 2) was not present after noncontact exposure to the BALB/c male. However, exposure to the female in diestrus produced significantly more cells in the rostral AOB than in the caudal AOB (asterisks: mitral layer, $t=$ 2.20; $\mathrm{df}=10 ; p=0.05$; granule layer, $t=2.56, \mathrm{df}=10 ; p=0.04) . b, c$, Photomicrographs showing c-fos immunoreactivity in the AOB of the ICR male after $2 \mathrm{hr}$ noncontact exposure to a BALB/c male $(b)$ or an ICR female in diestrus $(c)$. the distinct zones of cellular activation, we imposed a wire mesh barrier between the ICR males and the stimulus animal. The pair of animals remained in close proximity for $2 \mathrm{hr}$. No physical contact occurred, and it is unlikely that urine could have diffused through the bedding material to the other side of the barrier. The results are summarized in Figure 4. Noncontact exposure to the ICR female resulted in cellular activation that was similar in strength and pattern to that observed during contact exposure, indicating that the signals mediating this male-female interaction may be volatile. When separation was imposed between the ICR male and the BALB/c male, cellular activation was still present in the caudal AOB; however, it was accompanied by activation of the rostral AOB.

\section{DISCUSSION}

These results indicate that chemosensory signals associated with gender preferentially activate VRNs with cell bodies located in the apical part of the vomeronasal neuroepithelium and axons projecting to the rostral AOB. The caudal AOB, associated with VRNs located in the basal layers of the vomeronasal neuroepithelium, appears to respond preferentially to cues involved in interactions with foreign males. Our results indicate that the chemoarchitectural dichotomy of the peripheral vomeronasal system serves to segregate pheromone signals based on their behavioral context.

The pattern of activation in the rostral AOB of ICR males exposed to ICR females was the same in direct contact and barrier-separated conditions. This finding indicates that the chemosensory cues detected during this particular male-female interaction are not dependent on direct tactile contact with the female. Exposure of male mice to urine collected from females at different stages of the estrous cycle also results in preferential activation of the rostral AOB with higher numbers of activated cells observed during stages of the cycle associated with sexual heat (Dudley et al., 1999). Thus, female urine alone is a sufficient stimulus. Without tighter control over the possibility that some female urine could have passed through the barrier and directly assessed the male, we can only speculate that the urinary compound activating the rostral AOB is volatile in nature. Interestingly, a recent study demonstrated that volatile components of male rat urine applied to female rat VNO membranes resulted in the activation of $\mathrm{G}_{\mathrm{i}}$ proteins associated with $\mathrm{VRNs}$ projecting to the rostral AOB (Krieger et al., 1999).

Direct contact between foreign males produced cellular activation that was strictly confined to the caudal AOB and did not require the overt expression of aggressive behavior. According to the results of Krieger et al. (1999), the proteinaceous, presumably nonvolatile components of male urine stimulated the production of $G_{\alpha \mathrm{o}}$ in female rats, and $\mathrm{G}_{\alpha \mathrm{o}}$ is expressed in VRNs projecting to the caudal AOB. However, after placing a wire mesh barrier that prohibited direct contact between the two males, cellular activation was observed in both the rostral and the caudal AOB. If this pattern of activation is attributable solely to the nature of the pheromones involved, then recruitment of cellular activation in the rostral AOB in the noncontact situation suggests a scenario that seems to involve two types of pheromones. One pheromone (possibly volatile) involved in strain recognition activates the caudal AOB, whereas the other pheromone (possibly nonvolatile), involved in detection of a threat, causes inhibition of cells in the rostral AOB. On physical separation, in the absence of tactile cues and hence the elimination of the threat of overt aggressive behavior, the rostral inhibition is lost because the nonvolatile pheromone is no longer accessible, and activation is observed in both the rostral and caudal AOB. Alternatively, inhibitory actions may be provided by centrifugal afferents, such as those from the limbic system (Davis et al., 1978; Kevetter and Winans, 1981).

The distinct patterns of cellular activation observed in the $\mathrm{AOB}$ in the present study are likely to be the result of exposure to two different blends of pheromonal cues. In recent studies, the projections of VRNs from the apical zone expressing various subsets of the $\mathrm{G}_{\alpha \mathrm{i}}$ family of pheromone receptors were mapped to multiple glomeruli located almost exclusively in the rostral AOB (Belluscio et al., 1999; Rodriguez et al., 1999). Furthermore, one 
glomerulus was shown to receive input from more than one receptor subtype (Belluscio et al., 1999). These findings lend credence to the idea that complex pheromonal cues are organized at the level of the AOB into rostral and caudal compartments. Our results also suggest that a large degree of signal processing is performed at peripheral levels of the $\mathrm{VN}$ system. It can easily be visualized that a tight anatomical link between particular pheromone blends, spatially restricted regions of the AOB, and specific hypothalamic sites would help the animal to rapidly display the appropriate behavioral response to a given pheromone blend. The present demonstration that activation of distinct populations of neurons in the AOB can be detected in response to relatively gross chemosensory cues provides a bioassay that may be useful for identifying pheromone molecules processed by the VNO.

\section{REFERENCES}

Bean NJ, Wysocki CJ (1989) Vomeronasal organ removal and female mouse aggression: the role of experience. Physiol Behav 45:875-882.

Belluscio L, Koentges G, Axel R, Dulac C (1999) A map of pheromone receptor activation in the mammalian brain. Cell 97:209-220.

Berghard A, Buck LB (1996) Sensory transduction in vomeronasal neurons: evidence for $\mathrm{G}_{\alpha \mathrm{ao}}, \mathrm{G}_{\alpha \mathrm{ai}}$, and adenylyl cyclase II as major components of a pheromone signaling cascade. J Neurosci 16:909-918.

Bressler SC, Baum MJ (1996) Sex comparison of neuronal fos immunoreactivity in the rat vomeronasal projection circuit after chemosensory stimulation. Neuroscience 71:1063-1072.

Clancy AN, Coquelin A, Macrides F, Gorski RA, Noble EP (1984) Sexual behavior and aggression in male mice: involvement of the vomeronasal system. J Neurosci 4:2222-2229.

Coquelin A, Clancy A, Macrides F, Noble E, Gorski R (1984) Pheromonally induced release of luteinizing hormone in male mice: involvement of the vomeronasal system. J Neurosci 4:2230-2236.

Davis BJ, Macrides F, Youngs WM, Schneider SP, Rosese DL (1978) Efferents and centrifugal afferents of the main and accessory olfactory bulbs in the hamster. Brain Res Bull 3:59-72.

Døving KB, Trotier D (1998) Structure and function of the vomeronasal organ. J Exp Biol 201:2913-2925.

Dudley CA, Moss RL (1999) Activation of an anatomically distinct subpopulation of accessory olfactory bulb neurons by chemosensory stimulation. Neuroscience 91:1549-1556.

Dudley CA, Rajendren G, Moss RL (1992) Induction of FOS immunoreactivity in central accessory olfactory structures of the female rat following exposure to conspecific males. Mol Cell Neurosci 3:360-369.

Dudley CA, Kumar A, Moss RL (1999) Activation of an anatomically distinct subpopulation of neurones in the male mouse accessory olfactory bulb (AOB) following exposure to female mouse urine: effect of endocrine status. XXI Meeting of the Association for Chemoreception Sciences (Achems), Sarasota, Fl.
Dulac C, Axel R (1995) A novel family of genes encoding putative pheromone receptors in mammals. Cell 83:195-206.

Fernandez-Fewell GD, Meredith M (1994) c-FOS expression in vomeronasal pathways of mated or pheromone-stimulated male golden hamsters: contribution of vomeronasal sensory input and expression related to mating performance. J Neurosci 14:3643-3654.

Halpern M (1987) The organization and function of the vomeronasal system. Annu Rev Neurosci 10:325-362.

Halpern M, Shapiro RS, Jia C (1995) Differential localization of G proteins in the opossum vomeronasal system. Brain Res 677:157-161.

Herrada G, Dulac C (1997) A novel family of putative pheromone receptors in mammals with a topographically organized and sexually dimorphic distribution. Cell 90:763-773.

Jia C, Halpern M (1996) Subclasses of vomeronasal receptor neurons: differential expression of $G$ proteins $\left(G_{\mathrm{i} \alpha \mathrm{a} 2}\right.$ and $\left.\mathrm{G}_{\mathrm{o} \alpha \mathrm{a}}\right)$ and segregated projections to the accessory olfactory bulb. Brain Res 719:117-128.

Keverne EB (1983) Pheromonal influences on the endocrine regulation of reproduction. Trends Neurosci 6:381-384.

Kevetter GA, Winans SS (1981) Connections of the corticomedial amygdala in the golden hamster. I. Efferents of the vomeronasal amygdala. J Comp Neurol 197:81-98.

Krieger J, Schmitt A, Löbel D, Gudermann T, Schultz G, Breer H, Boekhoff I (1999) Selective activation of G-protein subtypes in the vomeronasal organ upon stimulation with urine-derived compounds. J Biol Chem 274:4655-4662.

Matsunami H, Buck L (1997) A mulitgene family encoding a diverse array of putative pheromone receptors in mammals. Cell 90:775-784.

Müller-Schwarze D (1983) Scent glands in mammals and their functions. In: Advances in the study of mammalian behaviours (Eisenberg JF, Kleiman DG, eds), pp 150. Provo, UT: American Society of Mammalogists.

Novotny M, Harvey S, Jemiolo B, Alberts J (1985) Synthetic pheromones that promote inter-male aggression in mice. Proc Natl Acad Sci USA 82:2059-2061.

Rodriguez I, Feinstein P, Mombaerts P (1999) Variable patterns of axonal projections of sensory neurons in the mouse vomeronasal system. Cell 97:199-208.

Ryba N, Tirindelli R (1997) A new multigene family of putative pheromone receptors. Neuron 19:371-379.

Vandenbergh JG (1994) Pheromones and mammalian reproduction. In: Physiology of reproduction (Knobil E, Neill JD, eds), pp 343-359. New York: Raven.

Wysocki CJ (1979) Neurobehavioral evidence for the involvement of the vomeronasal system in mammalian reproduction. Neurosci Biobehav Rev 3:301-341.

Wysocki CJ, Katz Y, Bernhard R (1983) Malivomeronasal organ mediates female-induced testosterone surges. Biol Reprod 28:917-922.

Yamazaki K, Yamaguchi M, Beauchamp GK, Bard J, Boyce EA, Thomas L (1981) Chemosensation: an aspect of uniqueness of the individual. In: Biochemistry of taste and olfaction (Cagen RH, Kare MR, eds), pp 85-92. New York: Academic. 